\title{
Path Restoration Schemes in GMPLS-controlled Translucent Networks
}

\author{
N. Sambo ${ }^{1}$, F. Cugini ${ }^{2}$, N. Andriolli ${ }^{1}$, A. Giorgetti ${ }^{1}$, L. Valcarenghi ${ }^{1}$, P. Castoldi ${ }^{1}$ \\ 1: Scuola Superiore Sant'Anna, Pisa, Italy \\ 2: CNIT, Pisa, Italy
}

\begin{abstract}
This paper investigates path restoration in translucent optical networks. Regenerator information is carried by GMPLS protocols (RSVP-TE signaling and OSPF-TE routing protocols). Restoration performance is evaluated in terms of blocking probability and control plane load. $^{1}$
\end{abstract}

Keywords: translucent network, GMPLS, restoration

\section{Introduction}

Translucent optical networks (i.e., optical networks with sparse opto-electronic regenerators) are a promising trade-off between opaque and transparent networks (i.e., networks equipped with all-electronic or all-optical switching devices respectively) [1]. On the one hand, the sparse usage of shared-per-node regenerators in translucent networks permits to overcome the signal degradation that affects transparent networks, fulfilling quality of transmission (QoT) requirements. On the other hand, network device costs are kept limited with respect to opaque networks. Many papers focused on regenerator placement and on static routing and wavelength assignment in translucent networks, as reviewed in [1]. In dynamic GMPLS-controlled translucent networks, the lightpath provisioning can take advantage either of OSPF-TE routing protocol or RSVP-TE signaling protocol. In the former case, the regenerator availability information (i.e., the number of available regenerators in a node) can be announced by an OSPF-TE node state advertisement (NSA), proposed in [2] for the advertisement of node capacity (e.g., wavelength converter) information. In the latter case, two dynamic lightpath provisioning methods for translucent networks exploiting RSVP-TE signaling protocol extensions are proposed in [3,4]. In particular, availability regenerator information is gathered in the traversed nodes during the lightpath set up. Then, this information is utilized for computing the route and for designating the regeneration candidate nodes.

However, as highlighted in [1], restoration in translucent networks has not been thoroughly investigated yet. In this paper, the restoration performance of GMPLS-controlled translucent networks is assessed. First, the study analyzes the path restoration blocking contributions in dynamic translucent networks. Then, two path restoration schemes, respectively based on RSVP-TE protocol alone and on both RSVP-TE and OSPF-TE protocols, are compared in terms of restoration blocking probability and control plane load.

\footnotetext{
${ }^{1}$ The work described in this paper was carried out with the support of the BONE-project ("Building the Future Optical Network in Europe"), a Network of Excellence funded by the European Commission through the 7th ICT-Framework Programme.
}

\section{Path Restoration schemes in GMPLS-controlled translucent networks}

The considered translucent network consists of $N$ nodes and $L$ bi-directional links. Each link supports $W$ wavelengths per direction. Each node consists of a photonic cross connect (PXC). $M \leq N$ nodes are equipped with a regeneration module composed of $r$ shared-per-node regenerators. The $M$ nodes are chosen with the signal quality prediction based regenerator placement algorithm (SQP) [5]. Each node maintains an up-to-date QoT parameter database (QPD) containing the QoT parameters characterizing the optical layer and a regenerator database (RD) containing the number of available regenerators-per-node. The considered QoT model (taking into account noise, chromatic dispersion, polarization mode dispersion and self phase modulation) has been detailed in $[3,4]$.

In GMPLS-controlled networks, in case of link failure, the node detecting the failure, e.g. the downstream node of the failed link, sends an RSVP-TE Notify message to the source nodes of all disrupted lightpaths. Then, each source node performs lightpath restoration. In this work, path restoration schemes that take into account physical impairments and regenerator information are considered. As in provisioning, source node $s$ computes a path to destination $d$ by applying the Constraint Shortest Path First algorithm to the network topology. Then, $s$ verifies whether the computed path has an acceptable QoT by consulting QPD. If no transparent path is available between $s$ and $d, s$ needs to require an opto-electronic regeneration at an intermediate node. Indeed, $s$ consults QPD and RD for computing a path and designating a regenerator-equipped node in order to partition the back up path into transparent segments. Then, $s$ starts signaling along the computed path. Two path restoration schemes are considered.

In the first scheme, called regeneration information based on signaling (RBS), the RSVP-TE signaling protocol carries, if necessary, the list of regeneration candidate node-ids. For this purpose, the Regenerator Object (RO) [4] is included in the RSVP-TE Path message. Upon reception of the Path message, each node listed in RO checks its regenerator availability. If no regenerator is available, the node replaces its node-id into RO with the node-id of the downstream neighbor. Otherwise, the node checks the QoT of the last transparent segment, i.e. the optical segment terminating at the same node. In case of unacceptable QoT, a RSVP-TE PathErr message is sent toward $s$, while in case of available regenerator and acceptable QoT of the last segment, the node forwards the Path message to $d$. At $d$, RO is copied into the RSVP-TE Resv message. Upon receiving the Resv message, each node listed in RO reserves regenerator resource for that lightpath. 
The second scheme, called regeneration availability advertisement (RAA), utilizes the same signaling scheme based on RO as in RBS. Moreover, RAA utilizes OSPF-TE regenerator information node state advertisement (RI-NSA) to advertise all network nodes about the number of available regenerators at a specific node. As in RBS, upon receiving the Resv message, if a node is listed in RO, it reserves regenerator resource for the lightpath. Moreover, with RAA the same node generates and floods an RI-NSA specifying its node-id and the number of available regenerators. Each node receiving the RI-NSA updates the corresponding RD entry with the number of available regenerators and node-id. In this way, each node is aware of the regenerator availability in the network. In both schemes when the Resv message reaches $s$, the lightpath is successfully restored.

\section{Simulation results}

The restoration performance is evaluated on a Pan European network topology with $\mathrm{N}=27, \mathrm{~L}=55, \mathrm{~W}=40, \mathrm{M}=7$, $r=8$. Lightpath provisioning requests are dynamically generated according to a Poisson process and uniformly distributed among all the source-destination pairs. Both inter-arrival and holding time are exponentially distributed with an average of $1 / \lambda=10^{4} s$ and $1 / \mu s$, respectively. It is assumed that during provisioning QPD and RD are updated by GMPLS protocols. Once in the steady state, a single link failure is randomly generated among all links.

Fig. 1 shows the overall restoration blocking probability and its contributions experienced by RBS and RAA as a function of $\lambda / \mu$. A lightpath restoration can be blocked for three reasons. The first contribution is due to lack of bandwidth (i.e., forward blocking) when no available bandwidth is found along the computed back up path. The second contribution is due to resource contention (i.e., backward blocking [6]), when concurrent RSVP-TE instances try to reserve the same wavelength $w$ in close time instants. In this case, only one Resv message actually reserves the selected wavelength. The third contribution is due to unacceptable QoT (i.e., QoT blocking), when no feasible chain of transparent segments along the back up path can be found, caused by lack of regenerators.

Forward blocking is not shown in Fig. 1 because it is negligible. Backward blocking increases with the offered network load because the number of concurrent reservation

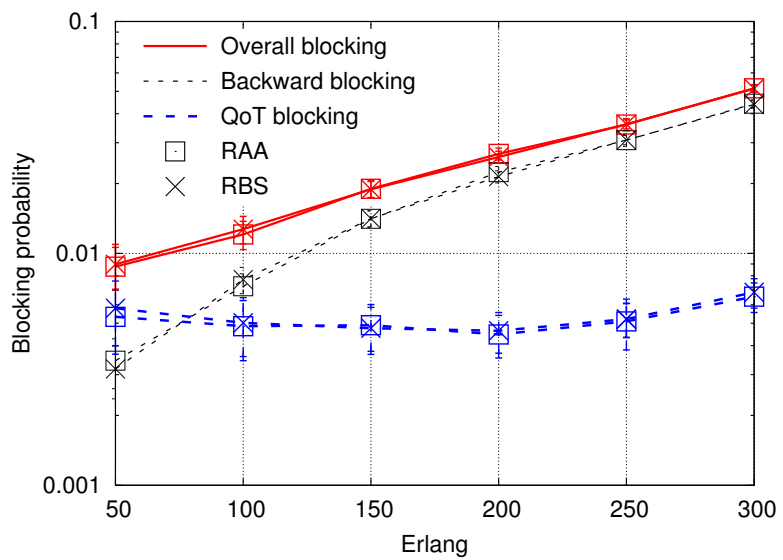

Figure 1: Restoration blocking probability experienced by RBS and RAA versus offered network load $(\lambda / \mu$ Erlang) instances increases as well. QoT blocking increases for high network load, due to lack of regenerators. RBS experiences almost the same overall restoration blocking probability than RAA. The similar backward blocking is expected because resource contention is not influenced by regenerator information. Even though the number of regenerators in the network is limited, the availability regenerator information flooded with RAA does not converge in due time, thus it does not succeed in decreasing QoT blocking. This happens because RI-NSAs are triggered during restoration when a Resv message reserves a regenerator resource. Typically, before that instant, the source nodes of the disrupted lightpaths have already computed the path, designated the regenerator nodes and started the restoration signaling. Fig. 2 shows the benefits of RBS in terms of control plane load during restoration. Indeed, the number of control plane packets (i.e., RSVP-TE messages and OSPF-TE RI-NSA) sent during restoration is much lower with RBS with respect to RAA since the former does not flood any RI-NSA.

\section{Conclusion}

This paper has investigated path restoration in translucent networks. Two path restoration schemes have been considered. RBS scheme exploits RSVP-TE signaling protocol, RAA scheme also exploits node state advertisement flooded by OSPF-TE routing protocol. Simulation results have shown that RBS experiences similar restoration blocking probability with respect to RAA requiring a much lower number of control plane packets.

\section{References}

[1] S. Gangxiang and R. Tucker, IEEE Comm. Mag., vol. 45, Feb. 2007

[2] G. Bernstein et al., Oct. 2007, Internet Draft, draft-bernsteinccamp-wavelength-switched-02.txt

[3] N. Sambo et al., Photonics in Switching 2007

[4] F. Cugini et al., ECOC 2007

[5] X. Yang and B. Ramamurthy, Photonic Network Communication, vol. 10, July 2005

[6] N. Sambo et al., IEEE Comm. Lett., vol. 11, Oct. 2007

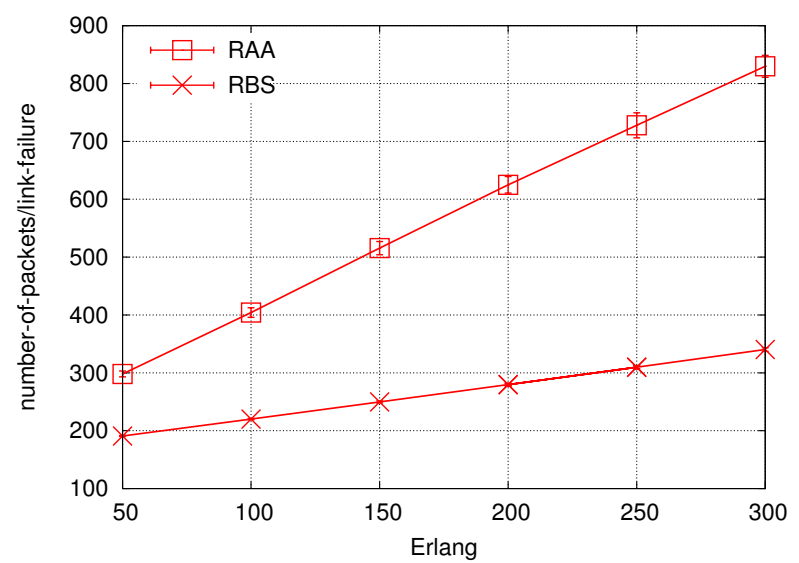

Figure 2: Number of control plane packets sent during restoration versus offered network load $(\lambda / \mu$ Erlang) 